\title{
TEORÍA Y RETOS DE LA EDUCACIÓN
}

\section{THEORY AND CHALLENGES OF EDUCATION}

\author{
Bertha Alice Naranjo Sánchez ${ }^{1}$ \\ Universidad Politécnica Salesiana, Ecuador
}

Este número de la revista Boletín Redipe aborda, por un lado, las reflexiones mesoaxiológicas del profesor Touriñán con relación al grado de penetración de la teoría de la educación en la Carrera de Pedagogía. Por otra parte está el tema de los retos de la educación, en el cual confluyen los demás artículos de investigación, incluido aquel: indagaciones que derivan en la formulación de retos en materia metodológica, didáctica, formativa, epistemológica, curricular, pedagógica y digital. Se reseña a continuación uno y otros trabajos.

\section{ALCANCE DE 'TEORÍA DE LA EDUCACIÓN' EN LA CARRERA DE PEDAGOGÍA.}

Artículo de investigación del pedagogo español José Manuel TOURIÑÁN LÓPEZ, Universidad de Santiago de Compostela, enfocado a entender el lugar de la teoría dentro del conocimiento de la educación, su diversidad

\section{Bertha Alice Naranjo Sanchez. Docente e} Investigadora de la Universidad Politécnica Salesiana Sede Guayaquil

https://orcid.org/0000-0002-4386-2335

bnaranjo@ups.edu.ec como disciplina y como investigación. Manifiesta que ello es posible

porque la teoría de la educación es conocimiento de la educación; hay una relación entre teoría y educación por medio del conocimiento de la educación. Cada modo de entender el conocimiento de la educación permite entender el significado de la teoría en relación con la 'educación'. Busca integrar las diversas posiciones que existen en la pluralidad semántica del concepto de "teoría" en relación con "educación", como disciplina y asignatura de plan de estudios y como investigación.

Respecto de la educación hay pluralidad de investigaciones teóricas: investigaciones teóricas acerca de la educación, investigaciones de teoría práctica, investigaciones teóricas de la educación (teoría como nivel de análisis epistemológico) e investigaciones de teoría de la educación como disciplina (pedagogía general).

En las disciplinas académicas sustantivas y en las asignaturas de planes de estudios 
están integradas por razones pragmáticas y por razones epistemológicas, según el caso, esas teorías. Las asignaturas pueden mezclar filosofía de la educación y teorías filosóficas cosmovisionarias y teorías interpretativas de la educación y teorías prácticas con teorías sustantivas de la educación (teoría como nivel de análisis epistemológico) y con pedagogía general (teoría como disciplina académica sustantiva), por razones pragmáticas, pero no deben conculcar las razones epistemológicas de la disciplina sustantiva en ningún caso.

\section{EL RETO DE EDUCAR EN TIEMPOS DE LA DIGITALIZACIÓN DE LA VIDA: HACIA UNA PEDAGOGÍA DE LAS RELACIONES ENTRE CUERPO, TEXTO Y TECNOLOGÍA.}

Artículo de investigación a cargo del académico colombiano Hernán Javier Riveros Solórzano, Universidad Distrital Francisco José de Caldas, Bogotá. Presenta una propuesta educativa para el desarrollo del pensamiento crítico en relación con los procesos de avance de la biotecnología y la digitalización de la vida. Para cumplir con este propósito se toma como punto de partida la relación entre cuerpo, texto y tecnología como un elemento de tipo estético y ético que permite comprender las diversas transformaciones de la existencia con el avance de diferentes herramientas en el ámbito de las tecnociencias y sus implicaciones en las transformaciones de la corporalidad y la subjetividad en su conexión con las condiciones sociales y culturales contemporáneas. Así mismo, a partir de un marco teórico y un diseño metodológico sintonizados con los debates actuales en el campo de los estudios sociales, educativos y tecnológicos, el documento expone las formas en las que sería factible consolidar acciones en el aula que permitan construir procesos pedagógicos, en clave de convergencia entre diversas disciplinas, para generar acciones educativas que respondan a las necesidades crítico - creativas de un contexto en el que la episteme de la vida como información exige de nuevas prácticas para pensar las formas de vivir y educar en una época de cambios y transformaciones como la contemporánea. Finalmente se presenta la biopragmática como posibilidad de trabajo educativo en el que se apuesta a una función crítica de la imaginación como elemento para proponer los procesos de apropiación de la existencia como obra de arte y del cuerpo como textualidad, los cuales son requeridos para asumir pedagógicamente un momento histórico transformado por condiciones como la aceleración del tiempo, la virtualidad del espacio y la racionalidad molecular propias del desarrollo de las tecnologías capaces de leer y modificar el código de la vida.

\section{LA NECESIDAD DE PROMOVER EL DEBATE EPISTEMOLÓGICO DE LA PEDAGOGÍA EN LAS FACULTADES DE CIENCIAS DE LA EDUCACIÓN, DESDE LA PERSPECTIVA} LATINOAMERICANA. Artículo de investigación elaborado por los académicos ecuatorianos Mirella Del Pilar Vera-Rojas, Unach, Luis Fernando Chávez Vera, Instituto Superior Tecnológico "San Gabriel" y Luis Antonio Vera Rojas, Espoch, Riobamba, Ecuador. Luego de hacer un breve recorrido histórico de la Educación, la Pedagogía y la escuela como organización social, expone las razones por las que el debate del estatuto epistemológico de la Pedagogía en América Latina debe ser revitalizado por educadores y pedagogos en las Facultades de Ciencias de la Educación, quienes además de formar integralmente a los educandos están llamados a continuar con el avance y desarrollo de esta ciencia, para empezar a educar a nuestros niños y jóvenes con modelos educativos y pedagógicos propios que respondan a las particularidades, singularidades, intereses y reales necesidades de la región, en función de nuestra particular cultura e historia, y refleje los avances ocurridos en los países que la conforman para brindar una educación emancipadora que tanto necesitan nuestros pueblos. 
ANÁLISIS DE FORMACIÓN MUSICALEN PROGRAMAS CURRICULARES DE EDUCACIÓN PARVULARIA CHILENA. Artículo de investigación autoría de los académicos chilenos Emanuel CerebelloGonzález; Eduardo Sandoval-Obando, Universidad SEK - Universidad Autónoma de Chile en torno al impacto positivo de la música en el desarrollo biopsicosocial del ser humano, asunto al que las instituciones académicas que forman a educadoras de párvulo le asignan escasa importancia en la formación de competencias musicales para el desempeño profesional. El trabajo expone la relevancia curricular de la educación musical, en las instituciones que forman educadoras de párvulo en Chile. Específicamente, se realiza una evaluación crítica en torno a las bases curriculares para la educación parvularia adoptando un diseño cuantitativo, descriptivo, transversal y no experimental.

\section{COMPLEJIZAR LA COMPETENCIA EN} LENGUAJE, UN TERRENO DE TRANSICIÓN ENTRE LO LINGÜÍSTICO Y LO MULTIMODAL, Artículo de investigación de Ernesto Fajardo Pascagaza, Camila Andrea Roa Rodríguez y Luis Carlos Cervantes Estrada. Analiza el concepto de competencia consignado en los Estándares Básicos de Competencias en Lenguaje, desde la óptica del pensador francés Edgar Morin (1968), reconocido filósofo, sociólogo y antropólogo. Para ello se discute desde una perspectiva contextualizada en las exigencias del nuevo y emergente ecosistema comunicativo. Esto exige un concepto de competencia transdisciplinar, renovada, y en proyección de la formación multidimensional del sujeto contemporáneo. En esta medida, el desarrollo de este nuevo tipo de competencia, requiere de la complejización de entornos, didácticas y metodologías a favor de la educación actual. Para tal efecto, se presenta el referente conceptual del pensamiento complejo, el lugar de la educación en este escenario, la compleja área dentro del currículo, y la transición entre competencias.
CARACTERIZACIÓN DE LOS AGENTES
EDUCATIVOS EN COLOMBIA. Artículo de investigación de los académicos colombianos Leidy Carolina Cuervo Alix Casadiego Cabrales Alvaro Avendaño Rodríguez y Luis Ferney Tovar Pérez Universidad Surcolombiana, Neiva $(\mathrm{H})$. Caracteriza los agentes educativos que atienden a la primera infancia en Colombia, de tal forma que se tenga una pauta inicial para diseñar planes de acción en torno a las directrices en materia de educación infantil. Concluye sobre la importancia de los aportes de la investigación para realizar capacitaciones adecuadas de acuerdo al perfil de los diferentes agentes educativos, y de esta manera lograr contribuir al desarrollo integral de la primera infancia.

DESAFÍOS DEL APRENDIZAJE DIGITAL PARA LA FORMULACIÓN DE PROYECTOS PEDAGÓGICOS EN LA AGROINDUSTRIA, Artículo de investigación adelantado por los académicos Jesús Alfonso Torres Ortega y Oscar Fernando Contento Rubio, Universidad La Salle, de Colombia. Manifiestan que Plantear soluciones adecuadas para contribuir en la construcción de conocimiento para el agro es una meta incluyente no sólo para las entidades gubernamentales sino un compromiso de las instituciones educativas involucradas con el campo. Para lograr este fin que busca la equidad en el campo y además mejorar la innovación para los agricultores, es primordial atender las brechas que hay entre el campo y la ciudad, y hacer que la sistematización llegue a sus manos de forma práctica y sencilla. Sin embargo, ¿Qué medidas toma el agro para la cuarta revolución industrial? Es forzoso refinar recursos educativos digitales orientados a apoyar las actividades agroindustriales en el campo empleando las herramientas que brindan las Tecnologías de la Información y la Comunicación (TICs) bajo la perspectiva de una economía circular. 
DESARROLLANDO HABILIDADES DE PENSAMIENTO CRÍTICO PARA MEJORAR LA COMPRENSIÓN LECTORA. Artículo de investigación elaborado por la académica ecuatoriana María Piedad RivadeneiraBarreiro, Universidad Técnica de Manabí, Ecuador. Orientado a determinar la relación entre las habilidades de pensamiento crítico y la comprensión lectora. Los resultados mostraron una relación insignificante entre las habilidades de pensamiento crítico y la comprensión lectora de los alumnos. Los hallazgos también revelaron que ambos grupos tenían pequeñas diferencias durante la prueba previa y posterior. La prueba previa y posterior también mostró cambios mínimos entre los grupos. La falta de concentración de los participantes en los textos, el desconocimiento y el escaso uso de sus habilidades de pensamiento crítico se evidenciaron en sus errores gramaticales, sintácticos y semánticos. Las implicaciones de estos hallazgos sugieren una mayor investigación en esta área, explorando las prácticas de enseñanza que fomenten las habilidades de pensamiento crítico y la comprensión lectora de los estudiantes.

\section{DESARROLLO DE COMPETENCIAS DIGITALES EN PROGRAMACIÓN DE} APLICACIONES MÓVILES EN ESTUDIANTES DE NOVENO GRADO A TRAVÉS DE TRES ESTRATEGIAS PEDAGÓGICAS. Artículo de investigación elaborado por los académicos colombianos Cristian Camilo Fonseca Barrera, Jorge Armando Niño Vega y Flavio Humberto Fernández Morales. Orientado a examinar el desarrollo competencias en programación de aplicaciones móviles en estudiantes de noveno grado. El principal resultado fue que los estudiantes aprendieron a programar aplicaciones para dispositivos móviles, a la vez que desarrollaban las competencias digitales, junto con una actitud de liderazgo e innovación. Se concluye con la necesidad de continuar implementando las TIC como estrategia pedagógica, ya que motivan a los estudiantes por aprender, a la vez que facilita la labor docente a la hora de enseñar.

\section{DESARROLLO DE CAPACIDADES EMPRENDEDORAS; UNA ALTERNATIVA} A TRAVÉS DE LA DIVERSIFICACION CURRICULAR. Artículo de investigación elaborado por los académicos peruanos Priscila Estelita Luján Vera - Marlon Martin Mogollón Taboada y Luis Ramón Trelles Pozo Universidad Nacional de Frontera. El trabajo consistió en elaborar una propuesta de diversificación curricular para el desarrollo de capacidades emprendedoras, sustentada en las teorías del emprendimiento, el trabajo y el currículo. Para lograr estos propósitos se identificaron los niveles alcanzados por el deficiente desarrollo de capacidades emprendedoras mediante el estudio de las dificultades para gestionar procesos de estudio de mercado, diseño, planificación, comercialización de bienes o servicios de uno o más puestos de trabajo de una especialidad ocupacional específica. Los resultados muestran que la mayoría de estudiantes no evidencia habilidades básicas para el desarrollo de emprendimientos y que necesitan ser instruidos para desarrollar capacidades emprendedoras.

\section{INTERNALIZACIÓN DE LA PAZ, MEDIANTE} LAS COMPETENCIAS SOCIOAFECTIVAS EN NIÑOS Y NIÑAS DE PREESCOLAR EN NEIVA. Artículo de investigación a cargo de los académicos colombianos Emperatriz Perdomo Cruz, Leidy Carolina Cuervo, Jenifer Lorena Barrera Monje y Eduardo Plazas Motta. Ponen de presente que conseguir la paz ha sido el propósito esencial de todo ser humano a nivel mundial, con los años los conflictos han aumentado en todos los ámbitos de la sociedad y la solución mediática es la violencia. La investigación se realizó en la Institución Educativa Enrique Olaya Herrera de la ciudad de Neiva, en la que participó toda la comunidad 
educativa. Los resultados abordaron importantes conclusiones y recomendaciones, se propuso empoderamiento conceptual, procedimental y actitudinal, se reconoció transformar las interacciones pedagógicas de los agentes. Se planteó construir relaciones menos violentas y solución pacífica de los conflictos e implementar un currículo experiencial fundamentado en la pedagogía invisible.

\section{OPTIMIZACIÓN ESTRUCTURAL Y ARQUITECTÓNICA DESDE LÓGICAS TOPOLÓGICAS A SOLUCIONES CONSTRUCTIVAS DE ARQUITECTURA EN TIERRA EN PELAYA CESAR. Artículo} de investigación a cargo de los académicos colombianos I M Cadena-Gonzalez, J A Delgado-Rojas, M Vergel-Ortega, Universidad Francisco de Paula Santander, Cúcuta, Colombia. Se centra en la problemática actual de los sistemas constructivos basados en acero y concreto con alto valor contaminante que han remplazado en gran medida el uso de materiales vernáculos como la tierra en procesos constructivos, subestimando por completo los factores de sostenibilidad que el material posee. El uso de sistemas sustentable se justifica por la necesidad de nuevos modelos de desarrollo sostenible en la construcción tras la crisis energética de la actualidad, donde a nivel mundial y nacional la tendencia de su uso es positiva pero regionalmente, el Cesar refleja poca apropiación del material, por lo que la investigación presenta como objetivo general el Aplicar lógicas de optimización topológica estructural y arquitectónica a la arquitectura en tierra en el municipio de Pelaya, Cesar, logrando determinar ventajas de las técnicas de arquitectura de tapia y bahareque para optimización topológica, con resultados que sugieren la intervención de estos modelos de diseño para generar sistemas constructivos que respondan a la normativa estructural de construcción colombiana articulados con sistemas constructivos en tierra con alto confort térmico.

\section{EL POSICIONAMIENTO DE MARCA EN UNIVERSIDADES COLOMBIANAS: UN DESAFÍO PARA LA EDUCACIÓN O ESTRATEGIA DE MERCADEO. Artículo} de reflexión propositiva de los académicos colombianos Nevis Balanta Castilla, Universidad Distrital Francisco José de Caldas, Luisa Stella Paz Montes, Universidad Francisco de Paula Santander y Diego Mauricio Álvarez Paz

Universidad El Bosque Colombia. Asumen que es indudable la importancia del tema de la marca en las culturas y especialmente para la colombiana, en la cual las marcas hacen parte de las preferencias cotidianas y se convierten en artefactos colectivos que permean diferentes ámbitos incluyendo el de la educación y la cultura. De este modo las marcas se constituyen en huellas léxico-semánticas que revelan muchos elementos de la memoria social y cultural en el mercado y contexto colombiano. La marca está directamente relacionada con lo que se quiere dar a conocer, con la imagen que se pretende crear y el posicionamiento buscado; esto hace diferenciar a una universidad pública o privada y sus servicios, de las demás, concebidas a veces como competidoras directas, indirectas $y$ de todas aquellas que necesariamente no son competidoras pero igualmente son participantes en un mercado que tiene cada vez menos estudiantes que se matriculan en educación superior. Todo ello plantea desafíos en la defensa de una marca o buen nombre y en la creación de estrategias de mercadeo educativo utilizadas por las instituciones para promocionar y promocionarse y para sobrevivir a la crisis actual de universidades privadas y a la disminución de estudiantes y deserción incluso en las públicas. 
OPTIMIZACIÓN DEL APRENDIZAJE DEL INGLÉS EN NIÑOS DE PRIMARIA CON EL USO DE DUOLINGO. Artículo de investigación de los colegas colombianos-Sebastián Bernal Rodríguez-y Astrid Ramírez Valencia' Universidad Distrital Francisco José de Caldas Bogotá Colombia. Dirigido a demostrar la incidencia de la inclusión de la plataforma Duolingo en una clase de inglés con niños de primaria dirigida por un docente en formación (practicante), quien se inquietó por saber por qué los niños presentaban muchas dificultades para asimilar una lengua extranjera, esto lo motivó a proponer dentro de su planeación de clase, concertado con el docente titular, la inclusión permanente de esta plataforma durante el desarrollo de su práctica docente, entonces con su ojo de investigador decidió indagar acerca del efecto del uso de Duolingo, durante las sesiones. Los resultados dejaron ver el incremento en el conocimiento de la gramática inglesa y el vocabulario, luego de la implementación de Duolingo en el aula de clase, demostrando una significativa mejoría académica, también se notó cambios de actitud y con ello de valores tendientes a la formación de un ser integral, en los educandos.

\section{LOS APODOS EN LAEDUCACIÓN SUPERIOR}

COLOMBIANA. Artículo de investigación autoría de la académica Nevis Balanta Castilla, UDFC, en torno a la construcción de imaginarios y representaciones sobre los docentes por parte de estudiantes universitarios, quienes mediante la metaforización expresan opiniones compartidas sobre el quehacer pedagógico y educativo de sus profesores e incluso sobre su comportamiento en la cotidianidad. Se ofrece además un repertorio de apodos de maestros colocados por jóvenes universitarios, quienes entre otras estrategias semánticas utilizan la metáfora como eje central para nombrar a sus profesores. El texto comprende además, elementos de tipo descriptivo y explicativo acerca de la acción de apodar en un contexto académico. Asimismo, se destacan los recursos sintácticos y semánticos de los que se vale el estudiante apodador para crear el nuevo nombre, y se habla de las motivaciones y características del apodo en su proceso de formación, un tema novedoso e interesante en el ámbito de la investigación educativa. La investigación es sociodialectal, pues se aborda una parte de la lengua y se intenta establecer relaciones entre lo lingüístico y lo sociocultural.

\section{ANALIZANDO A LOS ESTUDIOSOS DE LEONIDAS PROAÑO. Artículo de revisión a} cargo de los colegas ecuatorianos Ramos, Rómulo Arteño, Carretero Poblete Pedro Antonio $Y$ Vallejo Villalva Mónica Del Pilar, Universidad Nacional de Chimborazo, ECUADOR. Hace un recorrido de Monseñor Leonidas Proaño en torno al accionar tras la misión de Monseñor Leonidas Proaño, Obispo de Riobamba, lugar donde a pesar de haber transcurrido 109 años de su natalicio, su pensamiento y obras siguen latentes, no solo en la práctica viva del evangelio, sino en la enseñanza del valor, del respeto a la dignidad del ser humano, con un gran sentido de humildad, solidaridad e identidad, siendo parte de nuestra memoria, nuestras vidas y nuestros corazones. Mediante el análisis de textos y entrevistas los autores demuestran por qué se lo denomina el "Obispo de los pobres". En el análisis realizado se intenta captar el interés para que interesados en conocer y difundir su pensamiento tengan como herramienta de consulta.

\section{WEBQUEST HERRAMIENTA TECNOLÓGICA APLICADA LA ENSEÑANZA DEL INGLÉS.} Artículo de investigación de Laura del Pilar Moreno Pérez y Astrid Ramírez Valencia, UDFJC, en torno a la implementación de herramientas tecnológicas dentro del aula de clase para el desarrollo de actividades, usada por el docente para promover la participación, el aprendizaje cooperativo y la creatividad en los estudiantes. Expone las implicaciones del 
uso de Webquest en un grupo de niños de segundo de primaria en una escuela pública por cuenta de un practicante de inglés dando a evidencia los efectos de la implementación de los recursos tecnológicos en el proceso de enseñanza de inglés esta experiencia puede ser utilizada en cualquier otro ambiente pedagógico. Los resultados de este estudio, corroboran que el uso adecuado de las Webquest dentro de un ámbito de enseñanza es pertinente para el proceso educativo, además permite al docente innovar en sus clases, logrando que los estudiantes participen significativamente, dentro de la misma. 